\title{
A RECONSTRUCTION METHOD FOR BAND-LIMITED SIGNALS ON THE HYPERBOLIC PLANE
}

\author{
HANS FEICHTINGER AND ISAAC PESENSON
}

\begin{abstract}
A notion of band limited functions is considered in the case of the hyperbolic plane in its Poincare upper half-plane $\mathbb{H}$ realization. The concept of band-limitedness is based on the existence of the Helgason-Fourier transform on $\mathbb{H}$. An iterative algorithm is presented, which allows to reconstruct bandlimited functions from some countable sets of their values. It is shown that for sufficiently dense metric lattices a geometric rate of convergence can be guaranteed as long as the sampling density is high enough compared to the band-width of the sampled function.
\end{abstract}

\section{INTRODUCTION}

The main goal of the present article is to consider an iterative algorithm for reconstruction of band limited functions on the two-dimensional hyperbolic plane in its Poincare upper half-plane realization.

The notion of band limited functions plays a central role in the classical signal analysis in which signals propagate in Euclidean space. It seems interesting to extend this theory to other geometries in particular to hyperbolic spaces. In this connection we would like to mention very interesting investigations of A. Kempf [10, 11, who used our sampling theory on manifolds to develop an approach to quantization of space-time and information theory.

Analysis on the hyperbolic plane is also useful for other applied problems. As it was discovered by C. Berenstein and E. Casadio Tarabusi in 22, analysis on the hyperbolic plane plays an important role for electrical impedance imaging. Another interesting applications of the analysis on the hyperbolic plane to microwave technology were given by A. Terras in [25].

The classical sampling theorem says that if $f \in L^{2}(\mathbb{R})$ is from the Paley-Wiener space $P W_{\omega}, \omega>0$, i.e., if its Fourier transform $\hat{f}$ has $\operatorname{support} \operatorname{supp}(\hat{f})$ in $[-\omega, \omega]$, then $f$ is completely determined by its values at points $n \Omega$, where $\Omega=\pi / \omega$, i.e.,

$$
f(t)=\sum f(n \Omega) \frac{\sin (\omega(t-n \Omega))}{\omega(t-n \Omega)},
$$

where the equality holds in the $L_{2}$-sense. The Paley-Wiener theorem states that $f \in P W_{\omega}$ if and only if $f$ is an entire function of exponential type $\omega$.

The irregular sampling theory which was started by Paley and Wiener 14] was further developed by A. Beurling, P. Malliavin [3, 4], and H. Landau [13.

1991 Mathematics Subject Classification. 42C05; Secondary 41A17, 41A65, 43A85, 46C99 .

Key words and phrases. Poincare upper half-plane, Helgason-Fourier transform, convolutions, band-limited functions, Laplace-Beltrami operator. 
In the early 1990's, H. Feichtinger and K. Gröchenig have introduced iterative reconstruction methods (cf., the series of papers [5, 6], 7]) which allow to recover band-limited functions on $\mathbb{R}^{d}$ from their irregular samples.

On the other hand, a version of an irregular sampling theory in which bandlimited functions are reconstructed as limits of variational splines was developed by I. Pesenson in the case of $L_{2}\left(\mathbb{R}^{d}\right)[20$, in the case of stratified Lie groups [18, [19], in the case of manifolds [17, 21, 23, 24, and in the case of a general Hilbert space [22]. Note that the notion of band-limited functions in Hilbert spaces was developed by I. Pesenson in [12, [15] and [16] in connection with approximation theory and Besov spaces on manifolds.

In our recent paper [8, we developed an iterative algorithm for reconstruction of band limited functions on general Riemannian manifolds of bounded geometry. In the same paper we also proposed to specify our results for manifolds which are of interest for applications.

The present paper is organized as follows. In the second section we will briefly introduce Harmonic Analysis on the Poincare upper half plane $\mathbb{H}=S L(2, \mathbb{R}) / S O(2)$. In the third section we introduce our basic notion of band limited function by using a non-commutative Fourier analysis on $\mathbb{H}$ which is given by the Helgason- Fourier transform. In the last section we present our iterative reconstruction algorithm.

It is important to note that, due to the existence of rich harmonic analysis on $\mathbb{H}$, our description of band limited functions and our reconstruction method become much more constructive when compared to the case of a general Riemannian manifold.

\section{The Poincare upper half-Plane model of the hyperbolic plane}

Let $G=S L(2, \mathbb{R})$ be the special linear group of all $2 \times 2$ real matrices with determinant 1 and let $K=S O(2)$ be the group of all rotations of $\mathbb{R}^{2}$. The factor $\mathbb{H}=G / K$ is known as the 2-dimensional hyperbolic space and can be described in many different ways. In the present paper we consider the realization of $\mathbb{H}$ which is called Poincare upper half-plane (see [9], 25]).

As a Riemannian manifold, $\mathbb{H}$ is identified with the regular upper half-plane of the complex plane

$$
\mathbb{H}=\{x+i y \mid x, y \in \mathbb{R}, y>0\}
$$

with a new Riemannian metric

$$
d s^{2}=y^{-2}\left(d x^{2}+d y^{2}\right)
$$

and corresponding Riemannian measure

$$
d \mu=y^{-2} d x d y .
$$

If we define the action of $\sigma \in G$ on $z \in \mathbb{H}$ as a fractional linear transformation

$$
\sigma \cdot z=(a z+b) /(c z+d),
$$

then the metric $d s^{2}$ and the measure $d \mu$ are invariant under the action of $G$ on $\mathbb{H}$. The point $i=\sqrt{-1} \in \mathbb{H}$ is invariant for all $\sigma \in K$. The Haar measure $d g$ on $G$ can be normalized in a way that the following important formula holds true

$$
\int_{\mathbb{H}} f(z) y^{-2} d x d y=\int_{G} f(g \cdot i) d g .
$$


In the corresponding space of square integrable functions $L_{2}(G)$ with the inner product

$$
<f, h>=\int_{\mathbb{H}} f \bar{h} y^{-2} d x d y,
$$

we consider the Laplace-Beltrami operator

$$
\Delta=y^{2}\left(\partial_{x}^{2}+\partial_{y}^{2}\right)
$$

of the metric $d s^{2}$.

It is known that as an operator in $L_{2}(\mathbb{H})=L_{2}(\mathbb{H}, d \mu)$ which is initially defined on $C_{0}^{\infty}(\mathbb{H}), \Delta$ has a self-adjoint closure in $L_{2}(\mathbb{H})$.

Moreover, if $f$ and $\Delta f$ belong to $L_{2}(\mathbb{H})$, then

$$
<\Delta f, f>\leq-\frac{1}{4}\|f\|^{2}
$$

where $\|f\|$ denotes the $L_{2}(\mathbb{H})$ norm of $f$.

We can introduce the full scale of Sobolev spaces $H^{\sigma}=H^{\sigma}(\mathbb{H}), \sigma \in \mathbb{R}$. For $\sigma>0$ the space $H^{\sigma}$ is understood as the domain of the operator $(-\Delta)^{\sigma / 2}$ in the sense of the general theory of positive definite self-adjoint operators with the graph norm

$$
\|f\|_{\sigma}=\|f\|+\left\|\Delta^{\sigma / 2} f\right\| .
$$

For $\sigma<0, H^{\sigma}$ is understood as a dual space to $H^{-\sigma}$ with respect to the paring

$$
<f, h>=\int_{\mathbb{H}} f h d \mu .
$$

The Helgason-Fourier transform of $f$ is defined by the formula

$$
\hat{f}(s, \varphi)=\int_{\mathbb{H}} f(z) \overline{\operatorname{Im}\left(k_{\varphi} z\right)^{s}} y^{-2} d x d y,
$$

for $s \in \mathbb{C}, \varphi \in(0,2 \pi]$, and where $k_{\varphi} \in S O(2)$ is the rotation of $\mathbb{R}^{2}$ by angle $\varphi$.

We have the following inversion formula for all functions $f$ from the space $C_{0}^{\infty}(\mathbb{H})$ of infinitely differentiable functions on $\mathbb{H}$ with compact support:

$$
f(z)=\left(8 \pi^{2}\right)^{-1} \int_{t \in \mathbb{R}} \int_{0}^{2 \pi} \hat{f}(i t+1 / 2, \varphi) \operatorname{Im}\left(k_{\varphi} z\right)^{i t+1 / 2} t \tanh \pi t d \varphi d t .
$$

The Plancherel Theorem states that a map $f \rightarrow \hat{f}$ can be extended to an isometry of $L_{2}(\mathbb{H})$ (with respect to invariant measure $d \mu$ ) onto $L_{2}(\mathbb{R} \times(0,2 \pi]$ ) (with respect to the measure $\left.\left(8 \pi^{2}\right)^{-1} t \tanh \pi t d t d \varphi\right)$.

If $f$ is a function on $\mathbb{H}$ and $\varphi$ is a $K=S O(2)$-invariant function on $\mathbb{H}$ their convolution is defined by the formula

$$
f * \varphi(g \cdot i)=\int_{S L(2, \mathbb{R})} f\left(g u^{-1} \cdot i\right) \varphi(u) d u, i=\sqrt{-1},
$$

where $d u$ is the Haar measure on $S L(2, \mathbb{R})$. It is known, that for the HelgasonFourier transform the following formula holds true

$$
\widehat{f * \varphi}=\hat{f} \cdot \hat{\varphi} .
$$




\section{BAND LIMITED SIGNALS AND METRIC LATTICES}

In this section we introduce notions of band limited signals, metric lattices, develop some of their properties and prove inequalities which are used later.

Definition 1. We will say that $f \in L_{2}(\mathbb{H})$ is $\omega$-band limited function if $\hat{f}(s, \varphi)=0$, for $|s|>\omega$. The set of all $\omega$-band limited functions will be denoted as $E_{\omega}(\mathbb{H})$.

According to this variant of Harmonic Analysis on $\mathbb{H}$, the following formula holds:

$$
\widehat{\Delta f}=-\left(s^{2}+\frac{1}{4}\right) \hat{f} .
$$

This formula, the Plancherel theorem, and the Sobolev embedding theorem immediately imply the next result.

Theorem 3.1. For any real $\sigma$ and every $\omega \geq 0$ the set $E_{\omega}(\mathbb{H})$ is a closed invariant linear subspace of the Sobolev space $H^{\sigma}(\mathbb{H})$. If $f \in E_{\omega}(\mathbb{H})$ then, for every $\sigma \geq 0$, the function $\Delta^{\sigma} f$ belongs to $C^{\infty}(\mathbb{H})$ and is bounded on $\mathbb{H}$.

Moreover, the same formula (3.1) and the Plancherel theorem give us the following inequality (3.2), which is appropriate to call the Bernstein inequality.

Theorem 3.2. A function $f$ belongs to the space $E_{\omega}(\mathbb{H}), \omega \geq 0$, if and only if for every $\sigma \in \mathbb{R}$, the following inequality holds true

$$
\left\|\Delta^{\sigma} f\right\| \leq\left(\omega^{2}+\frac{1}{4}\right)^{\sigma}\|f\| .
$$

Proof. By using the Plancherel formula and (3.1) we obtain that for every $\omega$ - band limited function

$$
\left\|\Delta^{\sigma} f\right\|^{2}=\frac{1}{8 \pi^{2}} \int_{|t|<\omega} \int_{0}^{2 \pi}\left(t^{2}+\frac{1}{4}\right)^{\sigma}|\widehat{f}(t, \varphi)|^{2} t \tanh \pi t d \varphi d t \leq\left(\omega^{2}+\frac{1}{4}\right)^{\sigma}\|f\|^{2} .
$$

Conversely, if $f$ satisfies (3.2), then for any $\varepsilon>0$ and any $\sigma>0$ we have

$$
\begin{gathered}
\frac{1}{8 \pi^{2}} \int_{|t|<\omega+\varepsilon} \int_{0}^{2 \pi}|\hat{f}(t, \varphi)|^{2} t \tanh \pi t d \varphi d t \leq \\
\frac{1}{8 \pi^{2}} \int_{|t|<\omega+\varepsilon} \int_{0}^{2 \pi}\left(t^{2}+\frac{1}{4}\right)^{-2 \sigma}\left(t^{2}+\frac{1}{4}\right)^{2 \sigma}|\hat{f}(t, \varphi)|^{2} t \tanh \pi t d \varphi d t \leq \\
\left(\frac{\omega^{2}+\frac{1}{4}}{(\omega+\varepsilon)^{2}+\frac{1}{4}}\right)^{2 \sigma}\|f\|^{2} .
\end{gathered}
$$

It means that, for any $\varepsilon>0$, the function $\widehat{f}(t, \varphi)$ is zero on $(-\omega-\varepsilon, \omega+\varepsilon) \times(0,2 \pi]$. The statement is proved.

We take the point $i \in \mathbb{H}$ and consider a small open ball $B(i, r / 4)$ in the invariant metric $d s^{2}=y^{-2}\left(d x^{2}+d y^{2}\right)$. It is possible to find such elements $g_{j} \in G$ that the family of balls $B\left(x_{j}, r / 4\right), x_{j}=g_{j} \cdot i$, has the following maximal property: there is no ball in $\mathbb{H}$ of radius $r / 4$ which would have empty intersection with every ball from this family. Then the balls of double radius $B\left(x_{j}, r / 2\right)$ would form a cover of $\mathbb{H}$. Of course, the balls $B\left(x_{j}, r\right)$ will also form a cover of $\mathbb{H}$. Let us estimate the multiplicity of this cover. 
Note that the Riemannian volume $B(\rho)$ of a ball in $\mathbb{H}$ is independent of its center and is given by the formula

$$
B(\rho)=2 \pi \int_{0}^{\rho} \sinh t d t
$$

Every ball from the family $\left\{B\left(x_{\nu}, r\right)\right\}$ that has non-empty intersection with a particular ball $B\left(x_{\mu}, r\right)$ is contained in the ball $B\left(x_{\mu}, 3 r\right)$. Since any two balls from the family $\left\{B\left(x_{\nu}, r / 4\right)\right\}$ are disjoint, it gives the following estimate for the index of multiplicity of the cover $\left\{B\left(x_{\nu}, r\right)\right\}$ :

$$
\frac{B(3 r)}{B(r / 4)} \leq \frac{\int_{0}^{3 r} \sinh t d t}{\int_{0}^{r / 4} \sinh t d t} \leq(12)^{2} e^{3 r} .
$$

In fact, the last estimate can be improved.

We have proved the following Lemma.

Lemma 3.3. For any sufficiently small $r>0$ there exists a set of points $\left\{x_{j}\right\} \subset \mathbb{H}$, such that:

1) open balls $B\left(x_{j}, r / 4\right)$ are disjoint;

2) open balls $B\left(x_{j}, r / 2\right)$ form a cover of $\mathbb{H}$;

3) multiplicity of the cover by open balls $B\left(x_{j}, r\right)$ is not greater $N=N(\mathbb{H})=$ $12^{2} e^{3}$.

Definition 2. We will use notation $Z\left(\left\{x_{j}\right\}, r, N\right)$ for any set of points $\left\{x_{j}\right\} \in \mathbb{H}$ which satisfies the properties 1)- 3) from the Lemma 3.3 with a positive small $r$ and we will call such set a metric $(r, N)$-lattice of $\mathbb{H}$.

Let us emphasize that an $(r, N)$-lattice assumes a "uniform" distribution of points on $\mathbb{H}$ with respect to the hyperbolic metric. It will not look as a "uniform" distribution from the point of view of the Euclidean metric: it will become denser and denser when approaching the real line and will become sparser and sparser when going to infinity.

Sobolev spaces $H^{\sigma}(\mathbb{H}), \sigma \in \mathbb{R}$, can also be described in terms of local geodesic coordinates on $\mathbb{H}$.

In what follows we fix a small positive $r>0$ and an $(r, N)$-lattice in $\mathbb{H}$ which will be denoted by $Z\left(\left\{y_{\nu}\right\}, r, N\right)$. For the corresponding set of balls $\left\{B\left(y_{\nu}, r\right)\right\}$, which satisfy Lemma 3.3, we consider a uniformly bounded partition of unity $\psi=\left\{\psi_{\nu}\right\}$ associated with $\left\{B\left(y_{\nu}, r / 2\right)\right\}$. Namely, we give the following Definition.

Definition 3. A uniformly bounded partition of unity $\psi=\left\{\psi_{\nu}\right\}$ associated with $\left\{B\left(y_{\nu}, r / 2\right)\right\}$ is a set of non-negative $C^{\infty}(\mathbb{H})$ functions such that

a) $\operatorname{supp} \psi_{\nu} \subset B\left(y_{\nu}, r / 2\right)$,

b) $\sup _{x}\left|\partial^{|\alpha|} \psi_{\nu}\right| \leq C(\alpha)$, where $C(\alpha)$ is independent of $\nu$. 
An equivalent norm on $H^{\sigma}$ can be introduced by the formula

$$
\|f\|_{\sigma} \simeq\left(\sum_{\nu}\left\|\psi_{\nu} f\right\|_{H^{\sigma}\left(\mathbb{R}^{2}\right)}^{2}\right)^{1 / 2}
$$

where $\|f\|_{H^{\sigma}\left(\mathbb{R}^{2}\right)}$ denotes the regular Sobolev norm on the plane $\mathbb{R}^{2}$.

The following important result is an adaptation of the Lemma 3.3 from 21 for the case when the dimension of the manifold is $d=2$.

Theorem 3.4. For any $k>1$ there exist a constant $C=C(k, N)>0$ such that for any sufficiently small $r>0$ and any $(r, N)$-lattice $Z=Z\left(\left\{x_{\mu}\right\}, r, N\right)$ the following inequality holds true

$$
\|f\| \leq C\left\{r\left(\sum_{x_{j} \in Z}\left|f\left(x_{j}\right)\right|^{2}\right)^{1 / 2}+r^{k}\left\|\Delta^{k / 2} f\right\|\right\}, k>1 .
$$

\section{Iterative RECONSTRUCTION ALGORITHM}

In this section we describe our first iterative algorithms for reconstruction of band-limited functions on manifolds. The following Lemma 4.1 presents a generic idea which is used for reconstruction of different classes of analytic functions. This result basically says that if a bounded operator is close to identity operator then it can be inverted by a Neumann series.

The goal of section is to realize this idea in the case of band-limited functions on manifolds.

Lemma 4.1. Let $A$ be a bounded operator in a Hilbert space $H$ such that for some $\gamma<1$ and for all $f \in H$

$$
\|f-A f\| \leq \gamma\|f\| .
$$

Then $A$ is invertible and $f$ can be recovered from $A f$ by the following iterative procedure. If $f_{0}=$ Af and

$$
f_{n+1}=f_{n}+A\left(f-f_{n}\right)
$$

then

$$
\lim _{n \rightarrow \infty} f_{n}=f
$$

with the error estimate

$$
\left\|f-f_{n}\right\| \leq \gamma^{n+1}\|f\|
$$

Proof. Since the norm of the operator $I-A$ is less than 1, operator $A$ is invertible and, thus, its inverse $A^{-1}$ can be represented by a Neumann series $A^{-1}=\sum_{n=0}(I-$ $A)^{n}$. Thus, every $f \in H$ is determined by $A f$, because

$$
f=A^{-1} A f \sum_{n=0}^{\infty}(I-A)^{k} A f \lim _{n \rightarrow \infty}\left(\sum_{n=0}^{n}(I-A)^{n} A f\right) .
$$


The sequence $f_{n}$ of partial sums, $f_{n}: \sum_{k=0}^{n}(I-A)^{k} A f$, satisfies the stated recursion relation. Indeed,

$$
\begin{gathered}
f_{n+1} \sum_{k=0}^{n+1}(I-A)^{k} A f=A f+\sum_{k=1}^{n+1}(I-A) A f \\
A f+(I-A) \sum_{k=0}^{n}(I-A)^{k} A f=A f+(I-A) f_{n}=f_{n}+A\left(f-f_{n}\right) .
\end{gathered}
$$

Moreover, the identity

$$
\sum_{k=n+1}^{\infty}(I-A)^{k}=(I-A)^{n+1} A^{-1}
$$

implies that for all $f \in E_{\omega}(M)$ we have

$$
\left\|f-f_{n}\right\|=\left\|\sum_{k=n+1}^{\infty}(I-A)^{k} A f\right\|\left\|(I-A)^{n+1} A^{-1} A f\right\| \leq \gamma^{n+1}\|f\| .
$$

We will apply the above Lemma in the following situation.

Recall that in the previous section in the Definition 3 we fixed a uniformly bounded partition of unity $\left\{\psi_{\nu}\right\}$ subordinated to a family of balls $\left\{B\left(y_{\nu}, r / 2\right)\right\}$ and introduced Sobolev norms by the formula (3.5).

Consider an $(\varepsilon, N)$-lattice $Z\left(\left\{x_{j}\right\}, \varepsilon, N\right), \varepsilon \leq r / 2$, (see Definition 2). For an open cover by balls $\left\{B\left(x_{j}, \varepsilon / 2\right)\right\}$ satisfying Definition 2, we construct a uniformly bounded partition of unity $\left\{\theta_{j}\right\}$ subordinated to $\left\{B\left(x_{j}, \varepsilon / 2\right)\right\}$, with the properties:

a) $\operatorname{supp} \theta_{j} \subset B\left(x_{j}, \varepsilon / 2\right)$,

b) $\sup _{x}\left|\partial^{|\alpha|} \theta_{j}\right| \leq C(\alpha)$, where $C(\alpha)$ is independent of $j$.

Note that the assumptions that $\varepsilon \leq r / 2$ and that the multiplicity of the cover $\left\{B\left(y_{\nu}, r\right)\right\}$, which was used in the definition of the Sobolev norm (3.5), is not greater than $N=N(\mathbb{H})$, imply that each ball $B\left(x_{j}, \varepsilon / 2\right)$ has non-empty intersections with no more than $N(\mathbb{H})$ balls of the family $\left\{B\left(y_{\nu}, r / 2\right)\right\}$.

Given a function $f \in H^{k}(\mathbb{H}), k>1$, we consider an operator

$$
V_{Z, \theta}(f)=\sum_{j} f\left(x_{j}\right) \theta_{j}, \theta_{j} \in C_{0}^{\infty}\left(B\left(x_{j}, \varepsilon / 2\right)\right) .
$$

It will be shown that for any lattice $Z=Z\left(\left\{x_{\nu}\right\}, \varepsilon, N\right), \varepsilon \leq r / 2$, and corresponding uniformly bounded partition of unity $\theta=\left\{\theta_{j}\right\}$ subordinated to the cover $\left\{B\left(x_{j}, \varepsilon / 2\right)\right\}$, the function $V_{Z, \theta}(f)$ belongs to $L_{2}(\mathbb{H})$ as long as $f$ belongs to $H^{k}(\mathbb{H})$, where $k>1$.

To construct the operator $A$ we will need an orthogonal projection from $L_{2}(\mathbb{H})$ on the space of $\omega$-band limited functions $E_{\omega}(\mathbb{H})$.

We are going to describe this projection in terms of the Helgason-Fourier transform. In order to do so, we have to introduce an analog of the classical sinc function,

$$
\operatorname{sinc}(t)=\frac{\sin \pi t}{\pi t} .
$$


Definition 4. We definee the hyperbolic $\operatorname{sinch}_{\omega}(g), g \in S L(2, \mathbb{R})$, to be such a $S O(2)$-biinvariant function on $S L(2, \mathbb{R})$ whose Helgason-Fourier transform

$$
\widehat{\operatorname{sinch}}_{\omega}(s)
$$

is 1 for $|s| \leq \omega$, and 0 for $|s|>\omega$.

Now, we introduce the operator $P_{\omega}$ by the formula

$$
P_{\omega} f=f * \operatorname{sinch}_{\omega}, f \in L_{2}(\mathbb{H}) .
$$

It is clear that the operator

$$
P_{\omega}: L_{2}(\mathbb{H}) \rightarrow E_{\omega}(\mathbb{H})
$$

is the orthogonal projection on the subspace of $\omega$-band limited functions.

The operator $A$ will be defined as

$$
A_{Z, \theta} f=P_{\omega} V_{Z, \theta}(f),
$$

where $V_{Z, \theta}$ is defined in (4.6) and $P_{\omega}$ is the orthogonal projection from $L_{2}(\mathbb{H})$ onto $E_{\omega}(\mathbb{H})$.

The next goal is to provide a uniform estimate for the norms of the operator $I-A_{Z, \theta}$ on the subspace $E_{\omega}(\mathbb{H})$ for all $(\varepsilon, N)$-lattices with $\varepsilon \leq r / 2$.

The following lemma provides a step in this direction.

Lemma 4.2. For any lattice $Z=Z\left(\left\{x_{j}\right\}, \varepsilon, N\right), \varepsilon<r / 2$, and any uniformly bounded partition of unity $\theta=\left\{\theta_{j}\right\}$ subordinated to $B\left(x_{j}, \varepsilon / 2\right)$, the map $V_{Z, \theta}(f)$ is a continuous operator from $H^{k}(\mathbb{H}), k>1$, into $L_{2}(\mathbb{H})$. In other words, there exists a constant $C=C(\mathbb{H}, k)$, such that

$$
\left\|V_{Z, \theta}(f)\right\|_{L_{2}(\mathbb{H})} \leq C\|f\|_{H^{k}(\mathbb{H})},
$$

for all $f \in H^{k}(\mathbb{H})$.

Proof. According to (3.5) we have

$$
\left\|V_{Z, \theta}(f)\right\|_{L_{2}(\mathbb{H})}^{2} \sum_{\nu}\left\|\psi_{\nu} V_{Z, \theta} f\right\|_{L_{2}\left(B\left(y_{\nu}, r\right)\right)}^{2}
$$

and

$$
\left\|\psi_{\nu} V_{Z, \theta} f\right\|_{L_{2}\left(B\left(y_{\nu}, r\right)\right)}^{2} \leq\left\|\psi_{\nu} \sum_{j} f\left(x_{j}\right) \theta_{j}\right\|_{L_{2}\left(B\left(y_{\nu}, r\right)\right)}^{2} \leq C \varepsilon \sum_{j}\left|f\left(x_{j}\right)\right|^{2},
$$

where supp $\theta_{j}$ is in $B\left(x_{j}, \varepsilon / 2\right)$ and $C$ depends on the multiplicity $N=N(\mathbb{H})$. In other words,

$$
\left\|V_{Z, \theta}(f)\right\|_{L_{2}(\mathbb{H})} \leq C \varepsilon^{1 / 2}\left(\sum_{j}\left|f\left(x_{j}\right)\right|^{2}\right)^{1 / 2} .
$$

On the other hand, by a known inequality for $\mathbb{R}^{2}$,

$$
|f(y)| \leq C \sum_{0 \leq m \leq k} \varepsilon^{m-1}\|f\|_{H^{m}\left(B\left(x_{j}, \varepsilon\right)\right)}, k>1, C=C(k),
$$

where $y \in B\left(x_{j}, \varepsilon / 2\right), f \in C^{\infty}\left(B\left(x_{j}, \varepsilon\right)\right)$, we obtain

$$
\left|f\left(x_{j}\right)\right| \leq C \sup _{x \in B\left(x_{j}, \varepsilon / 2\right)}|f(x)| \leq C \varepsilon^{-1}\|f\|_{H^{k}\left(B\left(x_{j}, \varepsilon\right)\right)},
$$

where $k>1, C=C(k)$, and then 


$$
\left(\sum_{j}\left|f\left(x_{j}\right)\right|^{2}\right)^{1 / 2} \leq C \varepsilon^{-1}\left(\sum_{j}\|f\|_{H^{k}\left(B\left(x_{j}, \varepsilon\right)\right)}^{2}\right)^{1 / 2} .
$$

The last inequality and the inequality (4.10) give that there exists a constant $C$ which depends on smoothness $k$ and multiplicity $N(\mathbb{H})$, for which (4.9) holds true.

We will need the following Lemma 4.3 from 8 .

Lemma 4.3. For any $k>1$ there exist a constant $C=C(\mathbb{H}, k)>0$, such that for any small $0<\varepsilon<r / 2$ and any $Z\left(\left\{x_{j}\right\}, \varepsilon, N\right)$ the following inequality holds true

$$
\sum_{j}\left\|f-f\left(x_{j}\right)\right\|_{B\left(x_{j}, \varepsilon / 2\right)} \leq C \varepsilon\left\|(I+\Delta)^{k / 2} f\right\|, k>1 .
$$

Theorem 4.4. For a given $k>1$ there exist a constant $C=C(\mathbb{H}, k)>0$ such that for any lattice $Z\left(\left\{x_{j}\right\}, \varepsilon, N\right)$, with sufficiently small $\varepsilon>0$ and for any $f \in E_{\omega}(\mathbb{H})$

$$
\left\|f-A_{Z, \theta} f\right\| \leq C \varepsilon\left(1+\omega^{2}\right)^{k / 2}\|f\| .
$$

Consequently, for fixed $k>1$ and $\omega>0$ it is true that for any $\varepsilon>0$ satisfying

$$
\varepsilon<\left(C\left(1+\omega^{2}\right)^{k / 2}\right)^{-1},
$$

one has

$$
\left\|f-A_{Z, \theta} f\right\| \leq \gamma\|f\| \quad \text { where } \quad \gamma=C \varepsilon\left(1+\omega^{2}\right)^{k / 2}<1 .
$$

Proof. For any $f \in E_{\omega}(M)$ we have

$$
\left\|f-A_{Z, \theta} f\right\|=\left\|P_{\omega} f-P_{\omega} V_{Z, \theta}(f)\right\| \leq\left\|f-V_{Z, \theta}(f)\right\| .
$$

Next, since the following identity holds true

$$
f(x)=\sum_{j=j_{1}}^{j_{N_{M}}} \theta_{j}(x) f(x)
$$

we obtain

$$
\begin{gathered}
\left\|f-V_{X}(f)\right\| \leq\left\|f-\sum_{j} \theta_{j} f\left(x_{j}\right)\right\| \leq\left\|\sum_{j} \theta_{j} f-\sum_{j} \theta_{j} f\left(x_{j}\right)\right\| \leq \\
\sum_{j}\left\|f-f\left(x_{j}\right)\right\|_{L_{2}\left(B\left(x_{j}, \varepsilon / 2\right)\right.}
\end{gathered}
$$

where the sum can be estimated by using our inequality (4.11).

We have

$$
\left\|f-A_{Z, \theta} f\right\| \leq C \varepsilon\left\|(I+\Delta)^{k / 2} f\right\|,
$$

for any $k>1$.

Because, for $f \in E_{\omega}(\mathbb{H})$, the Bernstein inequality

$$
\left\|\Delta^{k} f\right\| \leq \omega^{2 k}\|f\|
$$

holds true, it yields the inequality

$$
\left\|f-A_{Z, \theta} f\right\| \leq C \varepsilon\left(1+\omega^{2}\right)^{k / 2}\|f\| .
$$


For a fixed $\omega>0$ and $k>1$, because $C=C(\mathbb{H}, k)$ depends only on $\mathbb{H}$ and $k$, we can choose

$$
\varepsilon<\left(C\left(1+\omega^{2}\right)^{k / 2}\right)^{-1},
$$

such that corresponding

$$
\gamma=C \varepsilon\left(1+\omega^{2}\right)^{k / 2}
$$

is less than 1. Theorem is proved.

Combining this result with Lemma 4.1 we obtain

Theorem 4.5. For a given $k>1$ and $\omega>0$ choose an $\varepsilon>0$ such that

$$
\varepsilon<(C)^{-1}\left(1+\omega^{2}\right)^{-k / 2},
$$

where the constant $C=C(\mathbb{H}, k)$ taken from Theorem 4.4. Then, for any $(\varepsilon, N)$ lattice $Z\left(\left\{x_{j}\right\}, \varepsilon, N\right)$ and for any corresponding operator $A=A_{Z, \theta}$ in the space $E_{\omega}(\mathbb{H})$ define inductively, starting from

$$
f_{0}=A f:
$$

$$
f_{n+1}=f_{n}+A\left(f-f_{n}\right) .
$$

Then in the space $L_{2}(\mathbb{H})$ the following convergence holds true

$$
\lim _{n \rightarrow \infty} f_{n}=f \text { in } L_{2}(\mathbb{H}),
$$

with the error estimate

$$
\text { and }\left\|f-f_{n}\right\| \leq \gamma^{n+1}\|f\|,
$$

where $\gamma=C \varepsilon\left(1+\omega^{2}\right)^{k / 2}<1$.

\section{REFERENCES}

[1] J. Benedetto, Irregular sampling and frames, Wavelets: A tutorial in Theory and Applications (C.K.Chui, ed.), Academic Press, Boston, 1992, pp. 445-507.

[2] C. Berenstein, E. Casadio Tarabusi, Integral geometry in hyperbolic spaces and electrical empendance tomography, SIAM J.Appl. Math. 56(1996), no.3, 755-764.

[3] A. Beurling, Local Harmonic analysis with some applications to differential operators, Some Recent Advances in the Basic Sciences, vol. 1, Belfer Grad. School Sci. Annu. Sci. Conf. Proc., A.Gelbart, ed., 1963-1964, 109-125.

[4] A. Beurling and P. Malliavin, On the closure of characters and the zeros of entire functions, Acta Math.,118, (1967), 79-95.

[5] H. Feichtinger and K. Gröchenig, Iterative reconstruction of multivariate band-limited functions from irregular sampling values, SIAM J. Math. Anal., 23, (1992), 244-261.

[6] H. Feichtinger and K. Gröchenig, Irregular sampling theorems and series expantions of bandlimited functions, Journ. Math. Anal. Appl., 167, (1992), 530-556.

[7] H. Feichtinger and K. Gröchenig, Theory and practice of irregular sampling. Wavelets: mathematics and applications, 305-363, Stud. Adv. Math., CRC, Boca Raton, FL, 1994.

[8] H. Feichtinger and I. Pesenson, Iterative recovery of band limited functions on manifolds, Contemp. Math., 2004, 137-153.

[9] S. Helgason, Differential Geometry and Symmetric Spaces,Academic, N.Y., 1962.

[10] A. Kempf, Aspects of information theory in curved space, Talk presented at 10-th Conf. Gen. Rel. and Rel. Astrophysics, Guelph,Canada, 28-31 May 2003, e-Print Archive: gr-qc/0306104.

[11] A. Kempf, A covariant ultraviolet cutoff in curved space-time, Phys.Rev.Lett. 92:221301, 2004.

[12] S. Krein, I. Pesenson, Interpolation Spaces and Approximation on Lie Groups, The Voronezh State University, Voronezh, 1990, (Russian).

[13] H. Landau, Necessary density conditions for sampling and interpolation of certain entire functions, Acta. Math., 117, (1967), 37-52. 
[14] R.E.A.C. Paley and N. Wiener, Fourier Transforms in the Complex Domain, Coll. Publ., 19, Providence: Amer. Math. Soc., (1934).

[15] I. Pesenson, The Best Approximation in a Representation Space of a Lie Group, Dokl. Acad. Nauk USSR, v. 302, No 5, pp. 1055-1059, (1988) (Engl. Transl. in Soviet Math. Dokl., v.38, No 2, pp. 384-388, 1989.)

[16] I. Pesenson, The Bernstein Inequality in the Space of Representation of Lie group, Dokl. Acad. Nauk USSR 313 (1990), 86-90; English transl. in Soviet Math. Dokl. 42 (1991).

[17] I. Pesenson, Lagrangian splines, Spectral Entire Functions and Shannon-Whittaker Theorem on Manifolds, Temple University Research Report 95-87, (1995), 1-28.

[18] I. Pesenson, Reconstruction of Paley-Wiener functions on the Heisenberg group, Amer. Math. Soc. Transl. (2) Vol. 184, (1998), 207- 216.

[19] I. Pesenson, Sampling of Paley-Wiener functions on stratified groups, J. of Fourier Analysis and Applications 4 (1998), 269-280.

[20] I. Pesenson, Reconstruction of band-limited functions in $L_{2}\left(R^{d}\right)$, Proceed. of AMS, Vol.127(12), (1999), 3593- 3600.

[21] I. Pesenson, A sampling theorem on homogeneous manifolds, Trans. of AMS, Vol. 352(9), (2000), 4257-4270.

[22] I. Pesenson, Sampling of Band limited vectors, J. of Fourier Analysis and Applications 7(1), (2001), 93-100.

[23] I. Pesenson, Poincare-type inequalities and reconstruction of Paley-Wiener functions on manifolds, J. of Geometric Analysis 14(1), (2004), 101-121.

[24] I. Pesenson, Deconvolution of band limited functions on symmetric spaces, will appear in the Houston J. of Math.

[25] A. Terras, Harmonic analysis on symmetric spaces and applications, Springer-Verlag, 1985.

Department of Mathematics, Vienna University, AUStria

E-mail address: hans.feichtinger@univie.ac.at

Department of Mathematics, Temple University, Philadelphia, PA19122

E-mail address: pesenson@math.temple.edu 